\title{
Grand Challenges in Vector-Borne Disease Control Targeting Vectors
}

\author{
Baldwyn Torto * and David P. Tchouassi* \\ International Centre of Insect Physiology and Ecology, Nairobi, Kenya
}

Keywords: vector-borne diseases, vector biology and ecology, vector adaptation, xenobiotics and vector evolution, climate and anthropogenic changes

The World Health Organization (WHO) predicts that the global population is expected to reach 9.8 billion in 2050 and projects growth mainly in global urban populations. The implications of this growth include changes in the urban environment, which will play an important role in public health, especially a significant proportion of global vector-borne tropical infectious diseases. Combined with these predicted developments is climate change and land use changes which will also greatly alter the tropical disease landscape and impact on public and veterinary health systems, especially of low-income countries. These factors will promote increasingly the convergence of humans, animal reservoirs of pathogens, and vector species to perpetuate the cycle of vector-borne diseases (VBDs) especially in the tropics.

Indeed $80 \%$ of the world's human population is at risk of one or more VBDs. Currently, approximately $20 \%$ of the global tropical infectious disease burden is vector-borne and this accounts for about 1 million human deaths annually, although the mortality rate in sub-Saharan Africa is disproportionately higher (1). The major global VBDs and those of focal importance can be caused by pathogens including parasites, viruses and bacteria vectored by mosquitoes (malaria, lymphatic filariasis, yellow fever, dengue, chikungunya, Rift Valley fever, West Nile fever, Zika virus disease, Japanese encephalitis); sand flies (leishmaniasis, Sandfly fever); blackflies (Onchocerciasis), fleas (Tungiasis, plague); lice (Typhus, Louse-borne relapsing fever); tsetse flies (human African trypanosomosis); triatome bugs (Chagas disease); ticks (Crimean-Congo hemorrhagic fever, tick-borne encephalitis, Lyme disease, Relapsing fever (borreliosis), Tularemia); aquatic snails (Schistosomiasis).

In the last 3 decades, previously controlled vector-borne diseases have resurged or remerged in new geographic areas, with some disease outbreaks affecting humans triggered by several novel pathogens (2). Few vaccines have been developed for preventive control of vector-borne diseases. Additionally, reports of resistance to drugs and insecticides by vector-borne pathogens and vectors, respectively, are increasing. Thus, there is an urgent need to develop new or complementary control tools for VBDs with vector control as a pillar to curb vector-human contact. Despite this recognition, underlying constraints associated with existing control measures against VBDs including vector adaptive strategies need to be addressed. Several priority research areas have been identified most of which target weak links in the pathogen-vector-host interactions. Examples include physical cues, chemical ecology, transmission blocking, vector adaptation including influence of xenobiotics on vector biology, and genetic control approaches.

Notably, during these three decades, an in-depth research to understand the mechanisms underpinning pathogen-vector-host interactions have provided new insights into both the biology of vectors and pathogens of VBDs. The role of physical cues such as vision, temperature, auditory, vibration, and humidity in vector biology has been well established. Chemical cues are also important for most vectors to exploit biological processes for their survival including plant feeding, host seeking, oviposition site selection, aggregation, mate finding and defense. The key 
drivers for such behaviors are olfaction and but also visual and contact chemical cues $(3,4)$. As novel and more sustainable and effective measures to controlling pathogen transmission are increasingly likely informed by vector bioecology, the role of all these cues in vector behavior need to be better understood, including elucidation of the sensory mechanisms at the cellular and molecular levels. More importantly, their integration to aid development of more effective vector reduction strategies: bait technologies for monitoring vector populations, next generation attractants, and repellents, toxicants in "attract and kill strategies", push-pull strategies combining repellents and attractants, to name a few, are important challenges for future research.

The insect's microbiome can impact vector competency significantly. Recent research has documented the presence of diverse species of endosymbionts and other microbiota in mosquitoes and other arthropods including spiroplasma and microsporidia, with the latter as a new target for transmission blocking of parasites in malaria mosquitoes (5). The use of vertically transmitted symbionts (from mother to offspring) is very attractive that can decrease the vectorial capacity of their vector hosts and could offer a more sustainable control strategy against VBDs. Already, the use of Wolbachia-based biocontrol against the arboviral disease dengue has been recommended for carefully planned pilot deployment under operational conditions (6). Future research is needed to elucidate the role of endosymbionts (and their metabolites) especially endemic strains in the transmission of pathogens associated with other disease vectors and their interaction with other key drivers of vector biology.

Vectors have had to adapt to the effects of climate change and human induced activities in ways to sustain or even drive the emergence and spread of many VBDs. For vector-borne zoonotic pathogens transmitted between animals and humans, anthropogenic changes can impact the abundance and distribution of animals with the potential to influence human disease risk. Understanding the factors that influence vector host-feeding patterns, their abundance and number of bites an infectious host receives are critical for estimating disease transmission potential (i.e. vectorial capacity) $(7,8)$. As part of risk assessment for emerging VBDs, studies of how changes in host abundance including humans and wildlife influence the vectorial capacity of disease vectors and potential to alter vector-borne disease transmission risk are also important challenges for future research. While acknowledging that VBD emergence involves a myriad of factors, integrated response systems are needed that link a better understanding of pathogen, host and vector diversity and dynamics to improve risk targeting, supporting early warning and improved response capacity. This improved response capacity will require investment in both institutional and individual capacities to adapt and respond to changing disease circumstances.

The effect of xenobiotics in the environment on vector evolution is of significance for improved control of VBDs with existing tools and worth urgent attention. These pollutants range from synthetic pesticides, toxins, heavy metals, including plant allelochemicals where exposure has been associated with tolerance or resistance to chemical insecticides $(9,10)$. Insecticide resistance constitutes an impediment and a threat to VBD control that largely rely on use of chemical insecticides targeting vector populations $(11,12)$. Tolerance to these pollutants has been ascribed to geographic range expansion of disease vectors and the diseases they transmit e.g. malaria. Also, these adaptive effects have been noted to drive evolution in disease vectors through changes occurring even at the genomic level $(9,10)$. Understanding the mechanisms that underlie xenobiotic tolerance at the molecular, physiological, behavioral levels will be crucial to devise ways to overcome pesticide resistance. The necessity to characterize impact of resistance on vector traits of relevance in pathogen transmission including survival, competence (13) will be interesting focal areas.

Among emerging strategies against VBDs is genetic control of vectors through gene drive technology. This approach seeks to suppress vector populations by introducing and spreading genes and traits of interest into and through vector populations, in a robust non-Mendelian inheritance fashion (14). Given advances in genetic manipulation technology such as Clustered regularly interspaced short palindromic repeats (CRISPR), assembled genetic constructs can be readily introduced into genomes, and preferentially transmitted across generations resulting in the rapid increase in the frequency of the genetic construct in populations of the target species $(14,15)$. Current gene drive targets few malaria vectors (Anopheles stephensi and An. gambiae) with successes mainly reported in laboratorybased studies. Advances in this burgeoning research area include the need to demonstrate efficacy in containment or field settings, consider the complexity of diverse vectorial landscape including detailed understanding of vector population genetic structure, ethical issues surrounding its use requiring stakeholder engagements to trigger and sustain critical conversations on potential risks for its use in vectorborne disease control especially in sub-Saharan Africa.

Traditional old approaches such as the Sterile Insect Technique (SIT), previously used to manage tsetse flies and certain agricultural pests, are being revived for use against mosquito disease vectors and under the auspice of area-wide integrated pest management programs. SIT can suppress, or in specific situations to eradicate existing vector populations and to prevent the establishment of disease outbreaks (16). In order to operationalize the use of the SIT, investments into further research are required with regards to handling, transport to the release location and actual release of sterile males, without causing significant impact to their survival or post-release performance, as well as a means to monitor their performance after release. Methods for population surveillance of male populations is also important so that the releases can be scaled to the target population, and to enable the progress, impact, and assessment of the SIT program.

Insecticide-based control of vectors will remain a cornerstone for controlling VBDs; however, sustainability will require enhanced stewardship regarding their use. We envision 
additional cutting-edge research informed by a deeper knowledge of the biology and ecology of vectors. These should allow for complementary arsenals to combat the growing VBD menace. Furthermore, advances in molecular detection systems will be invaluable to detect and characterize novel vector-borne pathogens as the need for improved modeling of VBD risk taking into consideration the ecological parameters of pathogen transmission.

\section{AUTHOR CONTRIBUTIONS}

BT and DPT contributed to manuscript conception and design, literature review, manuscript preparation, and critical review. All authors contributed to the article and approved the submitted version.

\section{REFERENCES}

1. Global vector control response 2017-2030. Geneva: World Health Organization (2017). Licence: CC BY-NC-SA 3.0 IGO.

2. National Academies of Sciences, Engineering, and Medicine. Global Health Impacts of Vector-Borne Diseases: Workshop Summary. Washington, DC: The National Academies Press (2016). doi: 10.17226/21792

3. Nyasembe VO, Tchouassi DP, Pirk CW, Sole CL, Torto B. Host plant forensics and olfactory-based detection in Afro-tropical mosquito disease vectors. PLoS Negl Trop Dis (2018) 12(2):e0006185. doi: 10.1371/journal.pntd.0006185

4. Tchouassi DP, Jacob JW, Ogola EO, Sang R, Torto B. Aedes vector-host olfactory interactions in sylvatic and domestic dengue transmission environments. Proc $R$ Soc B (2019) 286:20192136. doi: 10.1098/rspb.2019.2136

5. Herren JK, Mbaisi L, Mararo E, Makhulu EE, Mobegi VA, Butungi H, et al. A microsporidian impairs Plasmodium falciparum transmission in Anopheles arabiensis mosquitoes. Nat Commun (2020) 11:2187. doi: 10.1038/s41467020-16121-y

6. WHO. Mosquito (vector) control emergency response and preparedness for Zika virus. (2016). Available at: https://www.who.int/neglected_diseases/ news/mosquito_vector_control_response/en/.

7. LaDeau SL, Allan BF, Leisnham PT, Levy MZ. The ecological foundations of transmission potential and vector-borne disease in urban landscapes. Funct Ecol (2015) 29(7):889-901. doi: 10.1111/1365-2435.12487

8. Tchouassi DP, Torto B, Sang R, Riginos C, Ezenwa VO. Large herbivore loss has complex effects on mosquito ecology and vector-borne disease risk. Transbound Emerg Dis (2020) 00:1-11. doi: 10.1111/tbed.13918

9. Li X, Schuler MA, Berenbaum MR. Molecular mechanisms of metabolic resistance to synthetic and natural xenobiotics. Annu Rev Entomol (2007) 52:231-53. doi: 10.1146/annurev.ento.51.110104.151104

10. Kamdem C, Fouet C, Gamez S, White BJ. Pollutants and insecticides drive local adaptation in African malaria mosquitoes. Mol Biol Evol (2017) 34 (5):1261-75. doi: 10.1093/molbev/msx087

\section{FUNDING}

The authors gratefully acknowledge the financial support for this research by the following organizations and agencies: UK's Foreign, Commonwealth \& Development Office (FCDO); the Swedish International Development Cooperation Agency (Sida); the Swiss Agency for Development and Cooperation (SDC); the Federal Democratic Republic of Ethiopia; and the Government of the Republic of Kenya. The views expressed herein do not necessarily reflect the official opinion of the donors.

\section{ACKNOWLEDGMENTS}

We are thankful to the staff of the Behavioural and Chemical Ecology Unit, ICIPE, for logistical support.

11. Hemingway J, Ranson H. Insecticide resistance in insect vectors of human disease. Annu Rev Entomol (2000) 45:371-91. doi: 10.1146/annurev. ento.45.1.371

12. Ranson H, N'guessan R, Lines J, Moiroux N, Nkuni Z, Corbel V. Pyrethroid resistance in African anopheline mosquitoes: What are the implications for malaria control? Trends Parasitol (2011) 27(2):91-8. doi: 10.1016/j.pt. 2010.08.004

13. Thomas MB, Read AF. The threat (or not) of insecticide resistance for malaria control. Proc Natl Acad Sci (2016) 113(32):8900-2. doi: 10.1073/pnas. 1609889113

14. Ogaugwu CE, Agbo SO, Adekoya MA. CRISPR in sub-Saharan Africa: applications and education. Trends Biotechnol (2019) 37(3):234-7. doi: 10.1016/j.tibtech.2018.07.012

15. Teem JL, Ambali A, Glover B, Ouedraogo J, Makinde D, Roberts A. Problem formulation for gene drive mosquitoes designed to reduce malaria transmission in Africa: results from four regional consultations 2016-2018. Malaria J (2019) 18:347. doi: 10.1186/s12936-019-2978-5

16. Gouagna LC, Damiens D, Oliva CF, Boyer S, Goff GL, Brengues C, et al. Strategic approach, advances, and challenges in the development and application of the SIT for area-wide control of Aedes albopictus mosquitoes in Reunion Island. Insects (2020) 11(11):770. doi: 10.3390/insects11110770

Conflict of Interest: The authors declare that the research was conducted in the absence of any commercial or financial relationships that could be construed as a potential conflict of interest.

Copyright (C) 2021 Torto and Tchouassi. This is an open-access article distributed under the terms of the Creative Commons Attribution License (CC BY). The use, distribution or reproduction in other forums is permitted, provided the original author(s) and the copyright owner(s) are credited and that the original publication in this journal is cited, in accordance with accepted academic practice. No use, distribution or reproduction is permitted which does not comply with these terms. 